\title{
L'Homme
}

Revue française d'anthropologie

157| janvier-mars 2001

Représentations et temporalités

\section{Jean-Pierre Digard, Les Français et leurs animaux}

Paris, Fayard, 1999, 281 p., bibl., fig.

\section{Bernadette Lizet}

\section{CpenEdition}

Journals

Édition électronique

URL : http://journals.openedition.org//homme/5903

DOI : $10.4000 /$ lhomme.5903

ISSN : 1953-8103

Éditeur

Éditions de l'EHESS

\section{Édition imprimée}

Date de publication : 1 janvier 2001

Pagination : 327-332

ISBN : 2-7132-1357-6

ISSN : 0439-4216

Référence électronique

Bernadette Lizet, « Jean-Pierre Digard, Les Français et leurs animaux », L'Homme [En ligne], 157 | janviermars 2001, mis en ligne le 23 mai 2007, consulté le 21 septembre 2020. URL : http://

journals.openedition.org//homme/5903; DOI : https://doi.org/10.4000//homme.5903

Ce document a été généré automatiquement le 21 septembre 2020.

(c) École des hautes études en sciences sociales 


\section{Jean-Pierre Digard, Les Français et leurs animaux}

Paris, Fayard, 1999, 281 p., bibl., fig.

\section{Bernadette Lizet}

1 NEUF ANS après la publication de L'homme et les animaux domestiques. Anthropologie d'une passion $^{1}$, Jean-Pierre Digard reprend, approfondit et enrichit ce qui en constituait le chapitre XI : «Un cas parmi d'autres : le système domesticatoire européen ». Au vu de la liste des différents ouvrages rédigés (ou édités) par l'auteur, on pourrait ajouter qu'il revient sur un terrain initial, celui de «La vie pastorale à Bonneval-sur-Arc (HauteMaurienne) », publié en $1975^{2}$. Un singulier retour sur l'ethnologie de la France ; JeanPierre Digard l'accomplit en effet après un long détour par la monographie d'une ethnie de pasteurs nomades d'Iran, une série de directions (ou de co-directions) d'ouvrages sur le pastoralisme à travers le monde, sur la cuisine au Proche-Orient, sur le « fait ethnique » en Iran et en Afghanistan, sur l'équitation (publication des actes d'un colloque transdisciplinaires et interprofessionnel), et sur l'Iran moderne. L'anthropologie de la domestication constitue le point d'aboutissement de ce parcours. La hauteur de vues dont témoigne L'homme et les animaux domestiques - une synthèse sur l'action domesticatoire tenant compte de la diversité des cultures à travers l'histoire se révèle précieuse pour une analyse des liens qui unissent les Français à leurs animaux. L'une des caractéristiques de l'ouvrage (et de la démarche de l'auteur en général) consiste à jouer constamment sur les échelles d'analyse - ici et ailleurs -, et donc sur la distance : «l'ailleurs » permet de penser « ici », de donner sens et raison aux passions qui s'attachent aux pratiques et aux débats animaliers. Ainsi entend-il éclairer la question sensible et complexe des relations que les hommes entretiennent aujourd'hui avec leurs animaux, et celle des statuts respectifs de ces derniers, dont les flottements se reflètent dans une législation en pleine incohérence.

2 Le présent volume se compose de trois parties: "Attitudes", "Significations " et «Problèmes». La première se subdivise en cinq chapitres (« Le phénomène animal de compagnie », "Bétail et volaille : la plèbe animale», «Un animal intermédiaire : le cheval ", «La fascination du sauvage», «Des passionnés aux militants: le milieu 
animalier »). La deuxième en comporte trois («La construction de l'animal », «La construction du maître", "Culpabilité et rédemption»). La troisième traite de la frontière incertaine entre le sauvage et le domestique, des malentendus et paradoxes de la passion animalière, enfin des excès et dérives du militantisme animalier.

3 Jean-Pierre Digard expose ses vues dans une langue claire et concise. Il a enrichi son appareil de références bibliographiques générales sur la domestication - largement interdisciplinaires - par des sources spécifiques au terrain français. Des éléments d'enquêtes personnelles y ont été adjoints, ainsi que des informations chiffrées très éclairantes (par exemple, dans le chapitre sur le milieu animalier, on pourra se renseigner sur le nombre de races de chiens (400!), d'éleveurs, de chiens importés, ou sur le taux de fréquentations des salons et des expositions spécialisés, etc.). Des points de discussion critique concernant l'ouvrage précédent ont également été ajoutés. Une introduction très dense campe le décor théorique. La domestication des animaux est considérée comme un fait culturel (les relations que les hommes établissent avec l'animal sont variables selon les sociétés et les époques) et comme un système (" À chaque situation concrète correspond un système domesticatoire particulier, qui se compose de tout ce que l'homme investit dans la production et l'utilisation des animaux: en action technique, en organisation sociale, en pensée [...], en représentations, etc.»; p. 14). Le système occidental européen («un cas parmi d'autres ») repose sur un système d'opposition entre deux groupes: dans une société ébranlée par la perte des repères d'autorité et des valeurs humanistes, les bêtes de compagnie, "aimées à la folie ", envahissent la vie de famille et l'espace public dans une confusion grandissante d'identité avec les êtres humains, tandis que les bêtes de rente, maltraitées, sont maintenues cachées.

4 À travers cette analyse de la société française dans ses rapports avec le monde animal (sauvage, domestique, urbain, rural), Jean-Pierre Digard livre un combat. L'ouvrage s'ouvre et se conclut sur un engagement contre les mouvements d'idées " animalitaires", contre les "entrepreneurs de morale qui mettent en parallèle racisme et spécisme, droits de l'homme et droits des animaux» (p. 223). Considérant "le décalage entre le droit et les faits ", l'auteur établit un passage entre recherche théorique et recherche appliquée; c'est ainsi qu'il formule des recommandations relatives à un nouveau découpage entre les catégories du sauvage et du domestique, et à l'attribution d'un certificat de capacité pour acquérir des chiens de grande taille (famille des pit-bull).

5 L'ouvrage fournit ample matière à réflexion, fourmille d'informations précieuses, apporte un éclairage décapant sur des faits massifs et préoccupants : la puissance du marché d'aliments pour animaux (pp. 195-197), des lobbies de producteurs d'aliments qui manipulent la recherche à leur profit, la corrélation établie entre les passions animalières et l'exclusion sociale, la multiplication des situations où la force de la relation à l'animal va de pair avec un appauvrissement du lien social... L'analyse est particulièrement fouillée en ce qui concerne l'animal de compagnie, le tissu associatif et ses activités, les courants d'idées et les logiques d'action cristallisés autour de la législation nationale et internationale (droit des animaux et spécisme). L'évolution de la famille et la fonction de l'animal « délégué et substitut narcissique » font par ailleurs l'objet d'utiles mises au point (chap. VII et X).

6 L'animal de rente est par contre moins bien traité : l'auteur ne l'a pas suivi « dans la chaleur des étables» françaises comme il a traqué le bestiaire familier. Et si cette 
vigoureuse perspective a l'immense mérite de circonscrire les problèmes et de proposer un modèle interprétatif, elle présente néanmoins des limites en schématisant des situations que l'enquête détaillée - dans la synchronie et la diachronie - peut révéler fort complexes et soumises à transformations rapides. Afin d'expliciter cette remarque, je discuterai le schéma construit dans le chapitre III autour du cheval, lequel tendrait « de plus en plus à occuper [...] une position intermédiaire entre le groupe des animaux de rente dont [il] ne fait déjà presque plus partie (races de trait lourdes exceptées) et celui des animaux de compagnie, qu'[il] est en passe de rejoindre » (pp. 68-69). Proposition relativisée en ces termes dans les dernières lignes du même chapitre : «À l'inverse de ce qui se passe pour les chevaux de selle, dont les protecteurs croient pouvoir plaider pour moins d'utilisation, la survie des chevaux de trait passe leurs amis le savent bien - par la reprise d'activités traditionnelles (débardage, voirie urbaine) et la recherche de nouvelles utilisations (jeux, spectacles)» (p. 70). D'un côté, donc, se généraliserait un principe de non-utilisation du « selle »- notamment chez les jeunes cavalières, entraînant une régression du sport vers l'activité ludique (randonnées par exemple), voire le simple maternage (c'est le « cheval potager ») -, et, de l'autre, un principe de réutilisation du « trait ».

Or, l'histoire des usages sociaux des anciens compagnons de travail des charretiers et des cochers me semble fournir un contre-exemple réjouissant aux dérives animalitaires - dont Jean-Pierre Digard souligne à juste titre les dangers - et à l'idée d'une dissolution du lien social qui s'exprimerait par un maternage de l'animal. Je l'étudierai donc de plus près.

8 Cette histoire est scandée par deux mouvements de « relance » qui ont en commun une très forte volonté de donner une nouvelle utilité économique et sociale au cheval de trait, frappé d'anachronisme. Après un demi siècle de marginalisation de l'élevage de bêtes à concours qui prenait la forme d'un hobby désuet, la première relance de production des années 80 transforme le cheval de trait en bête à viande. Les Haras nationaux, voyant la survie des neuf races françaises dans la construction d'une filière spécialisée (élevage, mise en marché, distribution bouchère), mobilisent le dispositif technico-économique des plans État-régions. Particulièrement fort chez les " hommes de chevaux» de la grande administration nationale, le tabou sur la question hippophagique a donc provisoirement sauté. Ce déverrouillage (concevoir une politique ouvertement basée sur un débouché principal en boucherie) s'explique par une collaboration inédite avec la recherche zootechnique ${ }^{3}$ et un partenariat nouveau (et plutôt agité) avec les syndicats d'éleveurs. Ces derniers investissent enfin la structure nationale qui est censée les représenter depuis une trentaine d'années, à savoir la Fédération nationale chevaline, secteur spécialisé de la Fédération nationale des syndicats d'exploitants agricoles (FNSEA). Les éleveurs vont-ils se " professionnaliser » grâce à une production de viande rentable et modernisée, selon le modèle du «baby beef » (organisation de l'élevage et de la mise en marché de type industriel) ? En 1979, le processus s'amorce avec l'obtention d'un «jumelage» qui impose un quota d'abattage entre les chevaux français et étrangers. Dans certains berceaux de race (Bretons, Percherons, Comtois) s'élabore une véritable culture d'éleveurs « chevalins »", fiers de leur spécificité et de leur réussite économique. Exportateurs par wagons entiers de coûteux reproducteurs vers l'Italie, les Bretons prennent ainsi leur revanche sur les gens du Perche qui avaient fait fortune avec leurs ventes américaines un siècle plus tôt. De nouveaux foyers d'élevage apparaissent dans le Massif Central et les Pyrénées, où l'espèce chevaline vient hybrider le savoir-faire construit sur les bovins. Cette 
intégration bovins-chevaux dans une culture technique nouvelle redonne aux éleveurs un peu de leur superbe des années fondatrices des berceaux de race, lavant une série d'humiliations: la perte d'autonomie de leurs syndicats en raison d'une lente absorption par les Haras, et la mise à l'écart des dispositifs qui modernisaient l'élevage français dans le cadre de la loi sur l'élevage de $1966^{5}$. Ces folles années de la viande sont donc marquées par une mobilisation syndicale sans précédent et par l'engagement des Haras dans une politique originale bousculant les valeurs établies : la "chevaline", nom familièrement donné aux bouchers spécialisés, apparaît comme une contreculture de "l'équestre ", c'est-à-dire l'équitation savante et les chevaux qui en permettent la pratique, héritage des loisirs aristocratiques géré par l'administration des Haras. Cette communauté du « cheval lourd » (terme officialisé par décret en juillet 1976), qui regroupe éleveurs, syndicats de race et État, a su débloquer la question hippophagique, susciter l'organisation de la filière à l'échelle nationale et internationale, et motiver les consommateurs.

La dynamique créée autour du «lourd» a profité à l'ensemble des professions du cheval (courses et sports équestres), dont la vente à l'abattoir constituait jusqu'alors un sous-produit «honteux» (pour reprendre l'expression de François Poplin') et faiblement valorisé. Les éleveurs de lourds ont conquis de haute lutte ce statut d'animal de rente, au sens agricole du terme (une production moderne spécialisée et rentable). Mais le cheval lourd n'aura fait qu'un bref passage dans les circuits de la bête à viande. Le caractère franchement protectionniste de l'accord de jumelage obtenu à l'arraché en 1979 n'était pas compatible avec l'accélération du processus d'intégration économique européen (libre concurrence).

Les Haras nationaux ont très vite abandonné toute référence à la viande (on a même recommandé de ne plus prononcer le mot «lourd», trop fortement connoté, dans les bureaux parisiens du service central). En pleine fièvre de restructuration - la transformation d'un service public en Établissement public administratif (EPA), imposant une prise en compte des besoins des «socio-professionnels»-, les Haras répondent aux critiques d'autoritarisme élitaire en réin- vestissant le cheval de trait. Ils découvrent la communication et centrent leur campagne très grand public sur ce héros populaire retrouvé, pour lequel ils conçoivent des compétitions originales d'attelage. Soumises à de spectaculaires épreuves sportives, les "montagnes de viande » portent les couleurs et les emblèmes identitaires propres à chaque pays (vêtements, harnais, voitures, actions techniques) ${ }^{7}$. 


\begin{tabular}{|c|c|c|c|}
\hline $\begin{array}{l}\text { Cheval de service } \\
\text { (transversal) }\end{array}$ & $\begin{array}{l}\text { Cheval de trait } \\
\text { (agricole) }\end{array}$ & $\begin{array}{l}\text { Cheval lourd (animal de rente) } \\
\text { Cheval de travail (neo-rural) }\end{array}$ & $\begin{array}{l}\text { Cheval "utilise" } \\
\text { (rurbain) }\end{array}$ \\
\hline - 3 millions de chevaux & $\begin{array}{l}\text { - } 2 \text { millions de chevaux } \\
\text { en } 1940,500000 \text { en } \\
1970\end{array}$ & $\begin{array}{l}\text { - stabilisation des } \\
\text { effectifs }\end{array}$ & $\begin{array}{l}\text { - petite remontée } \\
\text { d'effectifs }\end{array}$ \\
\hline $\begin{array}{l}\text { - de l'agnicole } \\
\text { (production, première } \\
\text { utilisation) au rural et à } \\
\text { l'urbain, dans un large } \\
\text { éventail de situations }\end{array}$ & $\begin{array}{l}\text { - création des neuf } \\
\text { grandes races de chevaux } \\
\text { de trait }\end{array}$ & $\begin{array}{l}\text { - campagne de relance } \\
\text { bouchere par les HN: } \\
\text { entrée dans la chaine de } \\
\text { production et de com- } \\
\text { merciatisation de l"animal } \\
\text { de rente (bête à viande) }\end{array}$ & $\begin{array}{l}\text { - campagne de relance } \\
\text { d's. Utilisation » (sport et } \\
\text { loisir) débouchant sur la } \\
\text { professionnalisation (vivre } \\
\text { et travailler avec les } \\
\text { chevaux) }\end{array}$ \\
\hline $\begin{array}{l}\text { - pas de races officielles } \\
\text { (mais des types de } \\
\text { chevaux comespondant } \\
\text { aux usages) }\end{array}$ & $\begin{array}{l}\text { - repli culturel et socio- } \\
\text { logique sur les berceaux } \\
\text { de race et emprise } \\
\text { progressive des HN }\end{array}$ & $\begin{array}{l}\text { - réinvention du cheval } \\
\text { de service multi-usages } \\
\text { par les néo-ruraux }\end{array}$ & $\begin{array}{l}\text { - ère du \& tout } \\
\text { patrimoinew (sauvegarde } \\
\text { des races et des métiers } \\
\text { anciens) }\end{array}$ \\
\hline $\begin{array}{l}\text { - les Haras nationaux } \\
\text { sont hors champ }\end{array}$ & $\begin{array}{l}\text { - patrimonialisation (de } \\
\text { moins en moins utilisé, } \\
\text { le cheval de trait devient } \\
\text { un emblème de temoirs) }\end{array}$ & $\begin{array}{l}\text { - renforcement de la } \\
\text { patrimonialisation } \\
\text { (notion de bien public et } \\
\text { collectif) }\end{array}$ & $\begin{array}{l}\text { - réhabilitation des } \\
\text { fonctions urbaines } \\
\text { (stratégje du déve- } \\
\text { loppement durable) }\end{array}$ \\
\hline $\begin{array}{l}\text { - grande diversité des } \\
\text { cultures techniques } \\
\text { autour de futilisation de } \\
\text { l'animal (chaîne des } \\
\text { métiers et des cultures } \\
\text { professionnelles) }\end{array}$ & $\begin{array}{l}\text { - évacuation de la ville } \\
\text { (traction chevaline } \\
\text { anachronique et mal- } \\
\text { séante) }\end{array}$ & $\begin{array}{l}\text { - rediversification } \\
\text { culturelie et sociale }\end{array}$ & $\begin{array}{l}\text { - le cheval de trait est } \\
\text { au carrefour de réseaux } \\
\text { d'acteurs (diversification } \\
\text { agnicole, sport insertion } \\
\text { et réinsertion sociales, } \\
\text { services techniques des } \\
\text { villes) }\end{array}$ \\
\hline
\end{tabular}

11 L'effort demandé à ces grands chevaux a d'abord eu les champs de course et les terrains de concours pour théâtre. La "sportisation» a rapidement débouché sur une réhabilitation du cheval de travail, avec la ville comme territoire d'action privilégié. La thématique du travail avec ces chevaux-patrimoine dans l'espace public entre en résonnance avec de multiples préoccupations contemporaines cristallisées dans les politiques publiques du développement durable: fragilité de l'emploi, inégalité des chances devant l'embauche, volonté de contrôler les désordres écologiques et sociaux de l'urbanisation, préservation des richesses biologiques et culturelles, et d'une qualité de vie dont les campagnes anciennes seraient les garantes... La scène urbaine est particulièrement révélatrice de ce qui a fait la réussite incontestable de la relance dite «d'Utilisation»: un transfert de responsabilité de l'État au privé et des syndicats d'éleveurs au grand public en ce qui concerne la sauvegarde du patrimoine des races de trait.

12 Ces passions utilisatrices des anciens chevaux agricoles et industriels (transport des marchandises lourdes: trains de wagonnets dans les mines, halage le long des canaux, etc.) démentent l'interprétation totalisante qui est celle de Georges Vigarello et Bernard Leconte dans leur introduction au numéro de Communications consacré au «spectacle du sport $»^{8}$, interprétation selon laquelle la référence à une morale fondatrice ( la première caractéristique moderne de l'olympisme, c'est d'être une religion", disait Coubertin) aurait totalement disparu. Le spectacle du «traitement tonique pour les faibles " pourrait bien revêtir encore cette dimension d'idéal à atteindre, ce qu'atteste le terrain ethnologique9. Les faibles, ici, se trouvent être paradoxalement ces bêtes colossales qui peuvent déployer une force qu'il faut savoir canaliser - un spectacle garanti. Mais la chaîne de production de ces " éléphants de nos campagnes ", comme dit un acteur bourguignon, s'est fragilisée à l'extrême. Le retour spectaculaire de ces animaux marginalisés évoque toutes les marginalités contemporaines, celles de l'agricole, de l'exclusion sociale, du handicap. Du sport au 
travail reconquis, les nouveaux usages ont suscité, dès la phase pionnière néo-rurale, une grande effervescence associative. Les associations et leurs médias sont de bons observatoires du prolongement rurbain du processus de néo-ruralisation initial : le cheval de trait et la traction animale au tournant $\mathrm{du}_{\mathrm{III}}{ }^{\mathrm{e}}$ millénaire illustrent bien ces situations "d'innovation au nom de la tradition ${ }^{10}$. Les "passeurs et traducteurs" rurbains de la culture chevaline ont entrainé les acteurs traditionnels des berceaux de races et les néo-ruraux dans une hybridation généralisée des savoirs et des savoir-faire de la traction et de l'attelage qui ne fait pas l'économie de conflits parfois vifs. Gageons, avec Jean Viard ${ }^{11}$, que "cette renaissance des traditions n'est pas tournée vers le passé » et "qu'une France plurielle est en train de s'organiser [...] L'identité [...] de demain ne sera pas celle d'hier, elle naîtra d'un mélange entre des populations différentes ».

Ce qui reste incertain, c'est l'économie des tensions exercées par les sensibilités animalitaires sur cet inventif chantier de pratiques. Voilà une centaine d'années, sous la pression d'une communauté de protecteurs de l'animal qui commençait à se structurer à l'échelle internationale, les attelages de travail étaient stigmatisés : les hommes de métier (cochers et charretiers) étaient accusés d'exercer des violences socialement dangereuses; l'animal de travail était tourné en dérision au titre de l'anachronisme technique. Dans le même mouvement, c'est ce cheval de service qui était poussé vers l'abattoir, justifiant la création d'une nouvelle institution sociale, l'hippophagie et ses lieux de mise à mort spécialisés. L'hippophagie ne s'est jamais banalisée, et la puissance du sentiment « animalitaire » laisse mal augurer de l'avenir. Mais il est intéressant de constater que le retour du cheval de travail urbain s'accomplit sous le contrôle sourcilleux des amis des animaux, ce que les Français dans leur très grande majorité semblent être devenus. Le travail ordinaire des employés des Calèches du château de Versailles, la plus grande PME d'attelage de France, et sans doute d'Europe - une douzaine de voitures et une équipe d'une trentaine de personnes en haute saison-, est un exemple passionnant à suivre. Depuis le poste de direction jusqu'à la conduite des attelages et aux soins d'écurie, cette équipe, presque exclusivement féminine, a accompli un transfert du sport au travail (c'est la compagne du champion de France d'attelage à deux chevaux, croisés selle et trait, qui est à l'origine du projet, et les femmes «cochers-meneurs » viennent aussi de la petite compétition). Cette professionnalisation s'effectue dans une extraordinaire tension. Les femmes qui font travailler ces chevaux expriment un respect empreint de tendresse pour leurs partenaires à quatre jambes, mais aussi le désir de réussir dans cette expérience "phare ». La tâche est rude pour tous, et le public ne manque pas de s'émouvoir des encolures suantes et des allures fatiguées, hésitant entre la compassion et l'adhésion militante à l'entreprise de requalification du transport hippomobile, de travail retrouvé pour ces très jeunes filles (emploi-jeunes), et de sauvegarde des races et de l'animal patrimoine. Aucun droit à l'erreur. Les cochers de l'an 2000 s'organisent : en 1999, ils ont créé un syndicat national professionnel chargé, entre autres, d'instituer un diplôme qui garantisse une formation technique spécialisée. Le fonctionnement de la machine animale, le seuil et les signes de souffrance y occupent une place centrale.

14 En quoi réside cette « différence fondamentale entre les mondes du selle et du trait »? J'avancerai une hypothèse : le trait est plus «intermédiaire » que le selle. Non parce qu'il constitue un passage entre le groupe des animaux de rente et celui des bêtes de compagnie, mais parce que la mémoire des anciens usages ruraux et urbains ne s'est pas perdue, et que l'inquiétude qui s'est installée dans les esprits quant à l'état de la 
planète suscite des engagements patrimoniaux. Le choc culturel de la disparition de la traction animale au bénéfice du moteur thermique est encore présent dans les mémoires, et les Haras nationaux ont su faire entendre l'appel à une large réappropriation sociale de ce patrimoine. Le cheval de trait fait le lien entre les acteurs de la relance et les spectateurs, entre les générations qui l'ont connu et celles qui le découvrent, entre la ville, le péri-urbain et le rural, entre les filières de la diversification agricole, du sport et de l'insertion, enfin entre les politiques publiques et la formation professionnelle.

\section{NOTES}

1. Paris, Fayard, 1990 (« Le temps des sciences »).

2. Grenoble, Centre alpin et rhodanien d'ethnologie, 1975.

3. Celle-ci s'effectue au CEREOPA (Centre d'étude et de recherche sur l'économie et l'organisation des productions animales), mis en place en 1969 par l'Institut national agronomique. Ce centre possédait une cellule spécialisée sur le cheval (Études et recherches pédagogiques sur le cheval), englobée en 1994 dans l'Institut du cheval. 4. Véritablement stigmatisé aujourd'hui, cet adjectif désigne familièrement le boucher hippophagique.

5. L'élevage du cheval était en effet déjà établi sur des bases réglementaires (service public) par les Haras nationaux. Les berceaux productivistes (Bretagne, par exemple) reprochent aux Haras leur traditionalisme dans les techniques de la reproduction et leur lenteur à assimiler des méthodes depuis longtemps maîtrisées par les autres filières, comme l'insémination artificielle (bovins, ovins-caprins et porcins).

6. François Poplin, « Le cheval, viande honteuse ", Ethnozootechnie, 1992, 48 : 23-34.

7. La culture «trait » se distingue de la culture équestre par un ancrage dans les berceaux (aujourd'hui, on parlerait plutôt de terroirs) qui déterminerait la valeur des animaux. Le pur-sang des « hommes de chevaux » aurait au contraire pour caractéristique de conserver son excellence intrinsèque quel que soit l'endroit où l'exportation du système du turf en a diffusé l'élevage. Depuis le début du siècle, les sports équestres ont par ailleurs progressivement élaboré des normes relatives à la compétition et aux vêtements des cavaliers.

8. Communications, 1998, 67 : Spectacle du sport : 7.

9. On parle par exemple de «mystique de la route ». La « route » est une épreuve d'endurance attelée, par équipes et relais, inspirée des compétitions mises au point pour les chevaux de selle. Elle s'inscrit dans un territoire de référence, brode sur un motif de l'histoire locale et cherche à valoriser les races chevalines de trait, mais aussi les produits locaux (Route du poisson sur le parcours Boulogne-Paris créée en 1991, Route du vin et des écluses en 1992, du comté en 1996, etc.).

10. Cf. Christian Bromberger \& Denis Chevallier, s. dir., Carrières d'objets. Innovations et relances, Paris, Éditions de la Maison des sciences de l'homme, 1999 (« Ethnologie de la France », Cahier 13) : 12.

11. Jean Viard, « Entretien avec C. Weil », Le Nouvel Observateur, 1999, nº 1825 : 19. 